\title{
Improved Sustainability of Railway Systems: The Tesys Rail Project
}

\author{
A. Frilli ${ }^{1}$, E. Meli ${ }^{1}$, D. Nocciolini ${ }^{1}$, L. Pugi ${ }^{1}$, A. Rindi ${ }^{1}$, M. Ceraolo ${ }^{2}$ \\ and G. Lutzemberger ${ }^{2}$ \\ ${ }^{1}$ Department of Industrial Engineering \\ University of Florence, Italy \\ ${ }^{2}$ Department of Energy, Systems, Territory and Construction Engineering \\ University of Pisa, Italy
}

\begin{abstract}
While an important part of the total carbon dioxide emission is still caused by transport systems, the impact of railway systems is significantly lower with respect to the other ground and air concurrent systems. However, the current development trends will produce a significant improvement of the efficiency of cars and planes, thus reducing the sustainability advantages of railway systems. This paper presents research work, based on the activities of the TESYS Rail Project, deals with the optimization of railway energy consumptions in order to increase the efficiency of the system. From the simulation of railway systems, the aim of the activity is to investigate how the use of energy storage devices can really improve efficiency in different operating scenarios, and involving different train typologies. A further goal of the activity is the development of integrated design, simulation and optimization tools and the generation of a code that can be integrated in real time applications. Finally, the model proposed in this paper has been validated with a set of experimental results provided by an industrial partner.
\end{abstract}

Keywords: railway, longitudinal dynamics, regenerative braking, contact line, electrical substations, energy storage.

\section{Introduction}

According to data available in literature [1], more than $30 \%$ of the total $\mathrm{CO}_{2}$ emission of the European community is still caused by transport systems (see Figure 1). Currently the impact of railway systems in terms of ratio between equivalent emissions and transported load (usually expressed in $\mathrm{gCO}_{2} / \mathrm{pkm}$ ) is less than one third respect to the other ground and air concurrent systems. As a consequence, the sustainability of railway systems is widely recognized. However, as reported in Figure 2, it is 


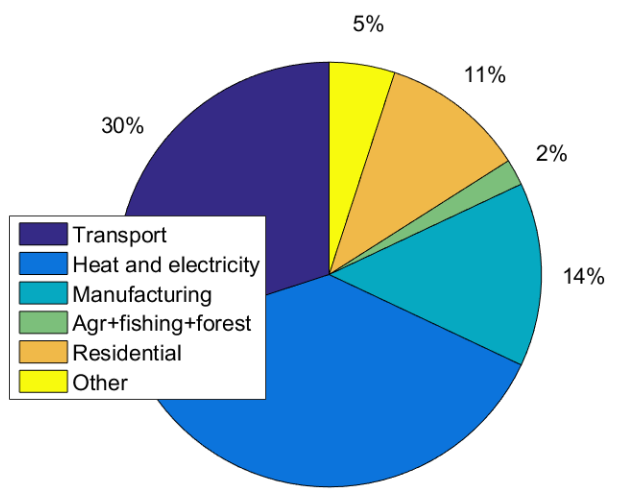

$38 \%$

(a)

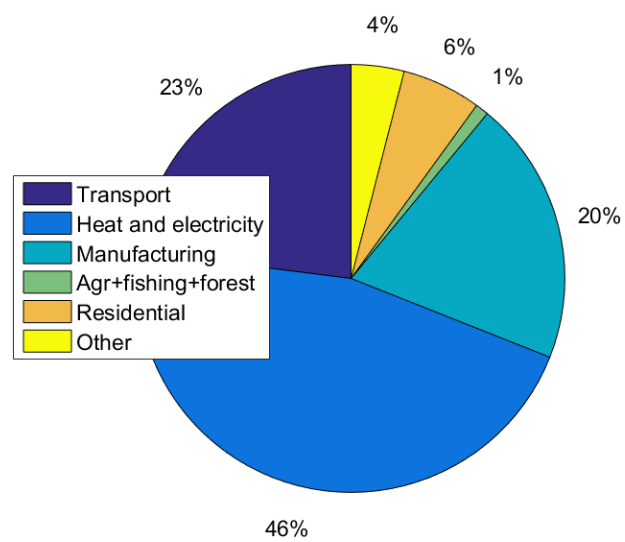

(b)

Figure 1: $\mathrm{CO}_{2}$ sources: (a) Europe, (b) world.

a common opinion that the technology trend will produce a significant improvement of the efficiency of cars and planes, estimating a reduction of the $\mathrm{CO}_{2}$ emissions of more than the $50 \%$ by the year 2050, thus strongly reducing the leadership of railway in terms of sustainability: railway will face the challenge of more efficient competitors and consequently it will have to undertake a strong improvement of the efficiency and sustainability of the whole system in order to meet the challenges of the future. Furthermore, the railway system has already undergone a critical phase of change for

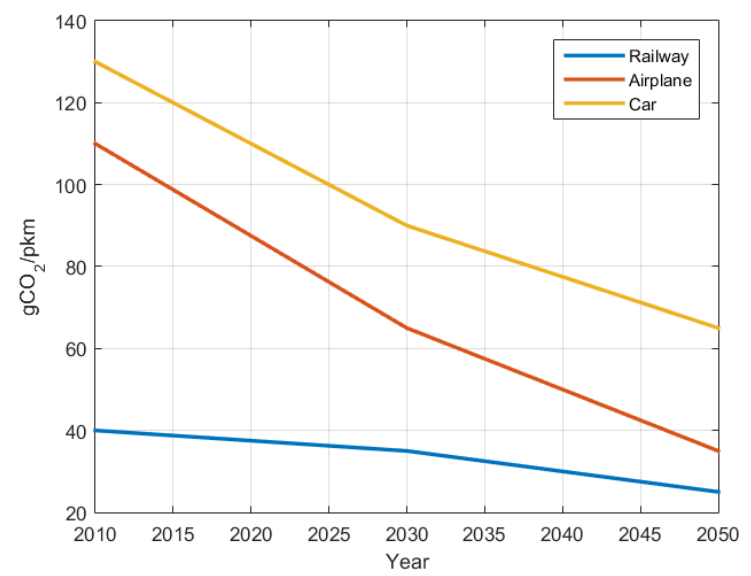

Figure 2: Comparison between the $\mathrm{CO}_{2}$ emissions of different transport methods.

the energy billing and management: the free energy market and the need for the vehicle to be interoperable between different energy infrastructures, managements and suppliers, considering also the presence of multiple carriers and railway operator have made it mandatory to be able to correctly calculate and measure the energy consumption of the vehicle and the losses due to the various elements of the vehicle-line system. An example of a system where different multiple stakeholders interact within a railway system is reported in Figure 3. The purpose of the Tesys Rail Project (acronym 


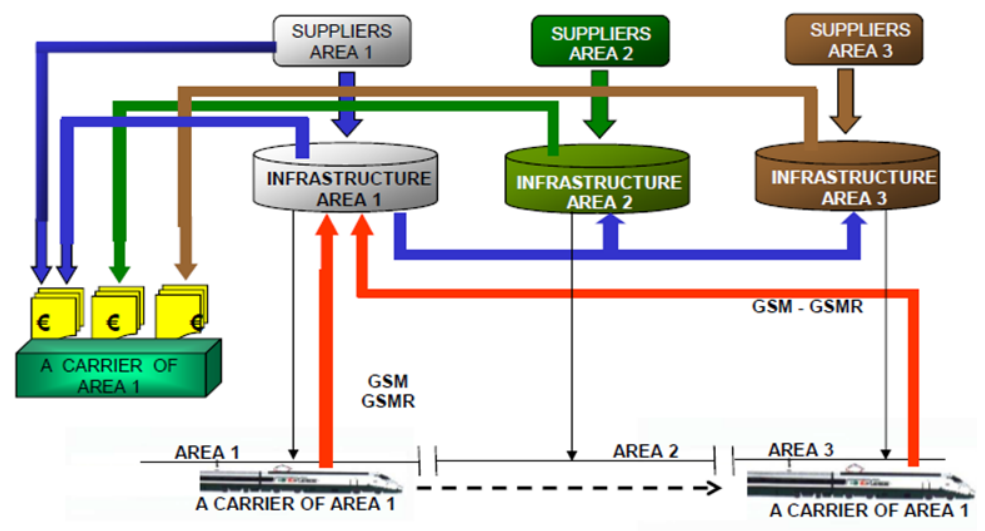

Figure 3: Energy billing and management.

of Methods and Instruments to improve environmental sustainability of railway systems) is to increase the sustainability of railway systems by joining the know-how of 8 major research and industrial partners such as Ansaldo STS, Thales, ATS PMI Toscana, Fondazione Politecnico di Milano, University of Florence, University of Naples. In particular this work deal with the activities coordinated by University of Florence, concerning the optimization of energy consumptions through the usage of energy storage systems in order to increase the efficiency of the whole system, recovering the regenerative braking energy, reducing power losses and avoiding problems due power peaks on the line. Figure 4 shows the possible energy recovery scenarios considered for the development of the proposed model: from a standard case without energy recovery it is possible to intervene adding stationary or on-board energy storage devices. In particular the work starts from a consolidated know-how [2], [3] dealing

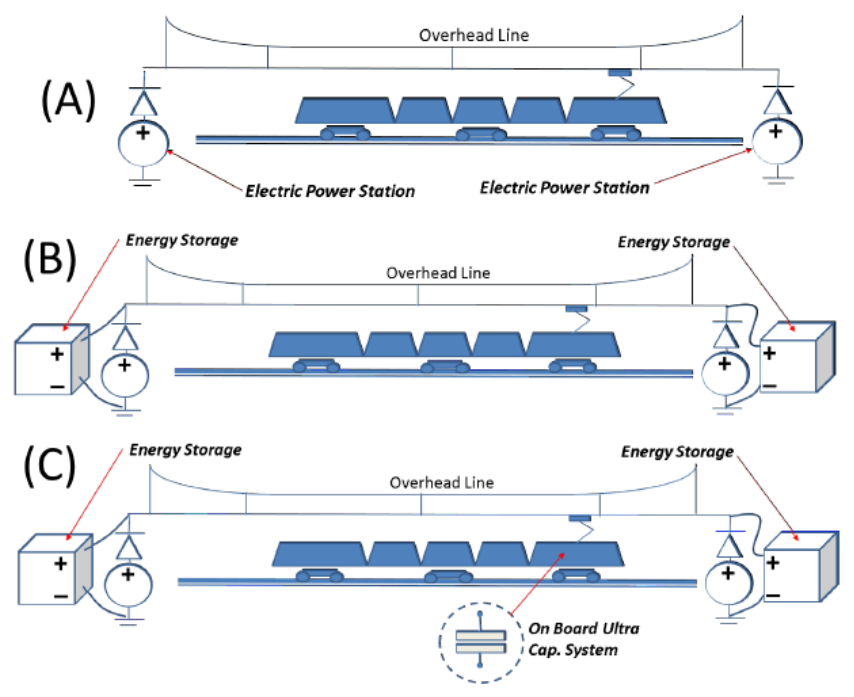

Figure 4: Energy storage scenarios.

with the energy optimization of tramways and the simulation of railway systems. The 
first purpose of the activity is to investigate how the usage of energy storage to recover energy can really improve efficiency in different complex operating scenarios, also involving different train typologies, such as high speed trains or more conventional railcars used for regional or urban transports. A second objective of the activity is the development of integrated design, simulation and optimization tools able to be easily shared from a restricted group of researchers to a wider community of research and industrial partners. The final objective of the activity is the generation of a code that can be integrated in real time applications devoted to the optimization of In this work proposed methodologies, tools and validation tests are introduced and explained respect to a population of benchmark tests. The core of the proposed model is based on the development of a computationally efficient model of the longitudinal dynamics of railway vehicle (see Figure 5); this model is devoted to the optimization of the system and of its mission profiles in terms of efficiency, sustainability and reliability. In particular, the model is able to reproduce the following phenomena:

- Longitudinal dynamics of the train: the mechanical behavior of the system is a result of the interactions between traction and braking forces and resistances to motion (both due to the vehicle and line induced and including the limitations that arise from limited wheel-rail adhesion);

- Power demand and energy flows: energy consumption and recovery, efficiency, calculated currents and corresponding interactions with the surrounding infrastructure.

The model has been developed in order to obtain the following characteristics:

- General and scalable approach: the model is able to reproduce a variety of different scenarios ranging from light railway and tramway systems to freight and passenger trains (including high speed trains);

- Modularity: the model is easily adaptable to different system configurations, allowing the analysis of different energy saving techniques;

- High level language: the use of a high level modeling environment allows an easier maintenance and usage for industrial and research applications;

- RT Implementation: the model can be used to produce code for real time applications like diagnostic, monitoring or control applications;

- Portability: the model is easily adaptable to different target/real time environment (different RTOS or even micro-controllers).

The development of efficient numerical models for the simulation and for the optimization of railway vehicle systems is an open research field and has a great industrial interest, as stated by recent contribution available in literature [2]. Most of these works [3]-[7], deal with the application of energy recovery and storage techniques in order to improve the efficiency of the system, reduce voltage and current fluctuations over 
the line and more generally improve the sustainability of the whole railway system. Most of the techniques used for energy recovery are commonly applied to tramways and light railway applications. In order to extend these techniques to conventional railways it is necessary to take into account in the train longitudinal dynamics analysis the variability of technical solution that can be adopted for the construction and the control of both traction and braking systems. Such variability is heavily influenced by the type of railway application (freight, conventional passenger or high speed trains). Furthermore, this type of analysis requires an accurate modeling of the braking phase and in particular of the blending between pneumatic braking, electric braking and adhesion independent device such as magnetic track system. Safety and operational issues (such as the distinction between service and emergency braking) also influence the complex interactions between different braking systems. In a previous work [2] the authors have focused on the detailed simulation of each sub-system, with a particular attention to some components such as the pneumatic [8] and electro-pneumatic [9] brake plant or the magnetic track brake [10], to which some specific studies have been dedicated.

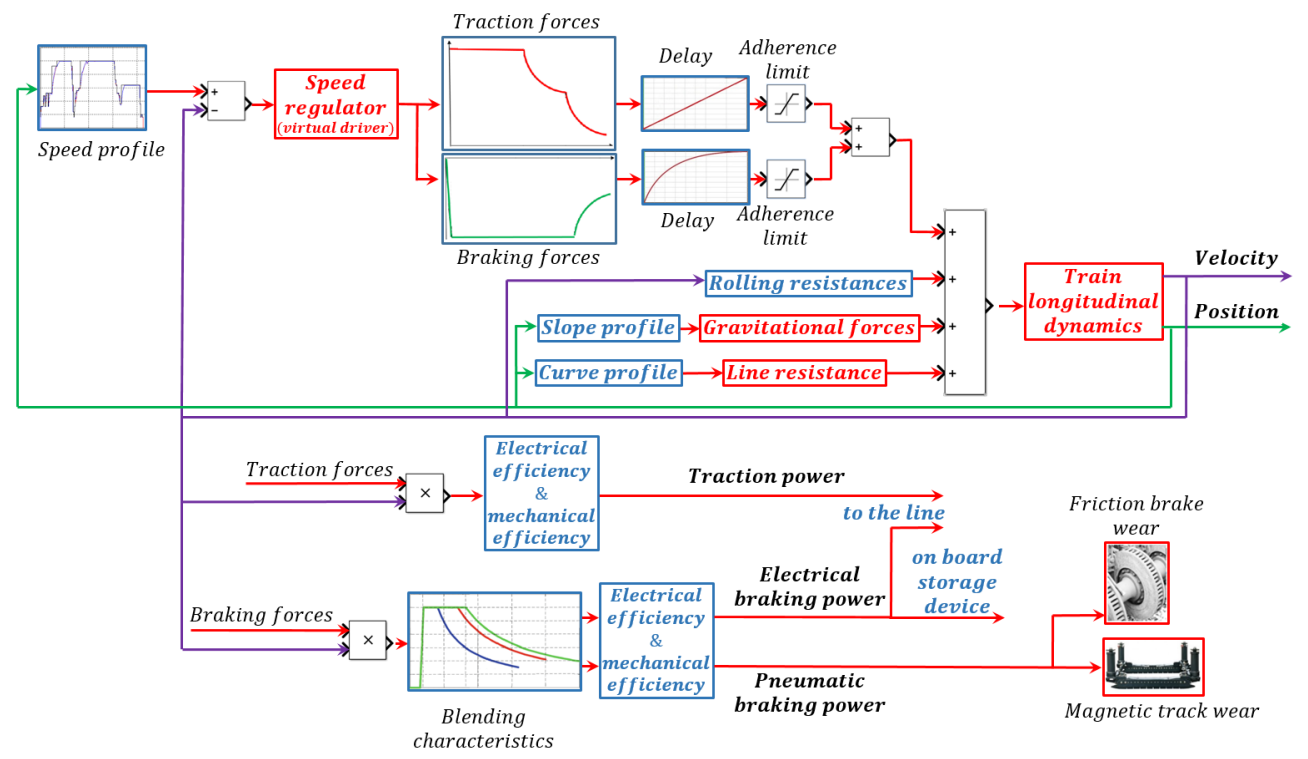

Figure 5: Architecture of the vehicle dynamical model.

\section{General architecture of the proposed model}

Two schemes of the elements of the proposed model are outlined in Figure 5 and in Figure 6: Figure 5 shows the architecture of the vehicle dynamical model and Figure 6 represents the interactions between the vehicle and the line. A position based speed profile is imposed to the train by a non-linear speed regulator (virtual driver) which controls the application of traction, coasting (no longitudinal effort applied) and braking (service or emergency braking according to the speed error and to the 
value of the safety state of the train). Traction effort, as in the model exposed in a previous work [2], is tabulated as a function of speed and line electrification standard, which is an infrastructure characteristic dependent on the train position. The traction and braking effort are dynamically applied to the train by using respectively a ramp signal and a delay. Furthermore, the longitudinal efforts are limited by the adherence condition of the line and by the distribution of traction and brake power on each wheelset (i.e. by the composition of the train). An innovative feature of the proposed modeling approach is the braking analysis, including the blending of pneumatic and regenerative electric systems: in order to develop a more general modeling tool, a TSI based (acronym of Technical Specifications for Interoperability) approach has been adopted. The TSI are the specifications by which each subsystem or part of subsystem is covered in order to meet the essential requirements and to ensure the interoperability of the European Community's high speed and conventional rail systems [17]. TSI [18]-[20] and the related EN standards and UIC fiches summarize and impose precise standard to which all the interoperable railway systems has to be constrained. Therefore, a TSI based modeling approach has major advantages in terms of availability of technical data (which are the same that the manufacturer has to produce for the homologation of the vehicle), generality and applicability. Since braking performances

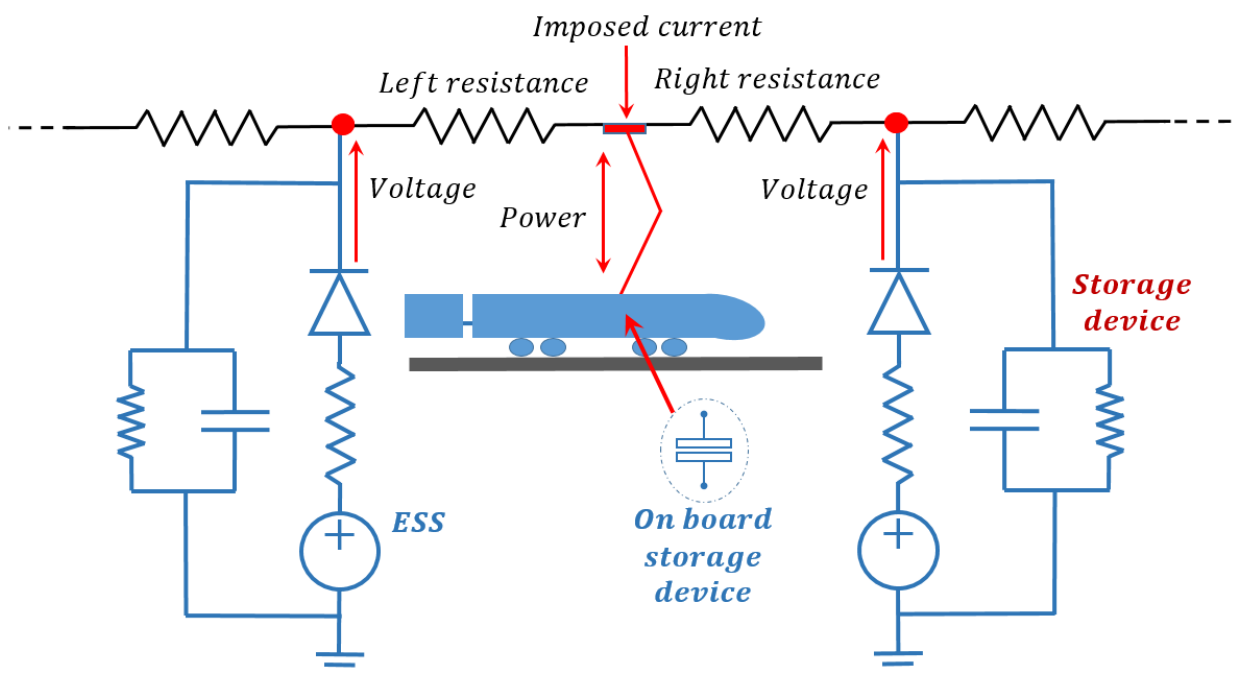

Figure 6: Scheme of the interactions between the vehicle and the line.

are strictly related to the safety of the railway system, the longitudinal braking effort is calculated as a tabulated function of speed, train mass and system state (service braking, emergency braking and optional degraded configurations). Braking performances are not related to the simulation of a specific system configuration (pneumatic or electro-pneumatic braking, magnetic track, electric braking) but to a brake power calculation set by UIC fiches [14], [15], EN standards [16] and TSI [17]-[20]. In order to satisfy these standards, safety specifications not only set limits on the braking performances but also constrain the design of the whole system. The blending policy and the distribution of braking power between subsystems (electrical and pneumatic) are calculated by a specific sub-component of the vehicle model (as visible in Figure 5) 
as a function of speed, line state (line voltage and electrification standard) and braking state (service braking, emergency braking, optional degraded configurations). If an on-board energy storage device is included in the system, its charging behavior can be analyzed as a function of three parameters: line voltage, state of charge and power available from the traction system. The conversion efficiency is evaluated through tabulated functions: for electrical components the efficiency depends on current and frequency while for mechanical components it depends on speed and power. The model of the line, outlined in Figure 6, has been developed using both standard and customized Matlab-Simscape elements. In particular, the line is modeled as a conductor fed by real voltage generators working in parallel to energy storage devices. The Balance based Bond-Graph approach used to model both vehicle and line elements represents an important innovation with respect to the approach exposed in previous works [2]: authors have abandoned the classical numerical approach of the previous Matlab Simulink implementation, in favour of a Matlab Simscape object oriented modeling [21], [22], [23]. An example of a simple Simscape model is reported in Fig-

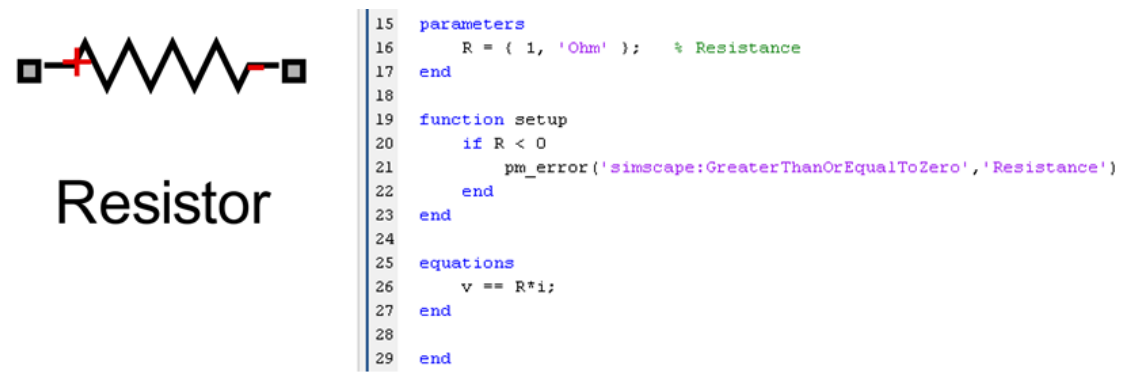

Figure 7: Example of object oriented Simscape block.

ure 7: each block is developed in terms of balance equations and each port represents a bidirectional connection which transmit variables associated to a physical domain. The new approach is better suited to assure modularity, maintainability and portability on different RTOS targets. For a preliminary validation of the results obtained with the proposed model, the high speed rail ETR 1000 on the direct route Florence-Rome has been considered (see Figures 12,8 and 13).

\section{Longitudinal dynamics}

The mechanical behaviour of the vehicle (i.e. its longitudinal dynamics) is simulated according to a quite efficient mono-dimensional model of the train which has been developed in previous research activities and described by the following Equation:

$$
F_{t}+F_{p}+F_{c}+F_{a}+F_{i}=0,
$$

Where:

- $F_{t}=\sum F_{t, i}$ is the sum of the longitudinal traction and braking efforts due to the train motorized axles; 
- $F_{p}=-m \cdot g \cdot \sin (\alpha)$ is the gravitational term due to the slope;

- $F_{c}=-m \cdot g \cdot i_{c}$ is the lumped contribution due to curves and line design;

- $F_{i}=-m \cdot \ddot{x}$ is the inertial term;

- $F_{a}=-m_{g} \cdot\left(a \cdot v^{2}+b \cdot v+c\right)$ is the rolling resistance term.

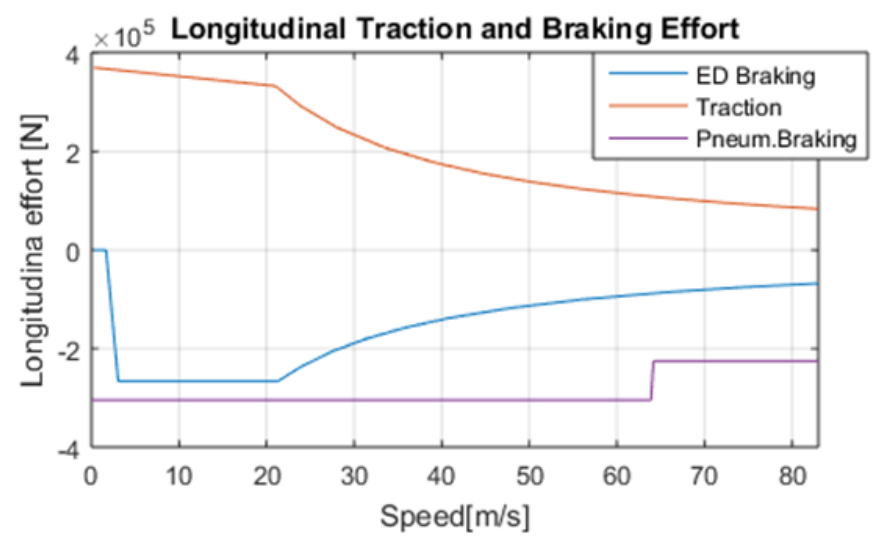

Figure 8: Traction and braking performances of the considered vehicle.

As reported in Figure 8, the traction and braking performances of the vehicle are function of the vehicle speed. In particular, the traction performance is characterized by three different trends:

- A first part where the traction effort is constant;

- A second part where the traction effort trend is hyperbolic and the vehicle power is constant;

- A last part where the vehicle power is inversely proportional to speed.

For trains that can be fed by different electrification standards, it is possible to define more than one traction-braking performance curve. Furthermore, it is useful to define those curves also for degraded adhesion condition; this is especially important for the braking efforts.

Figure 9 shows two possible blending strategy for the braking phase: the first case is the blending according to the performance of a TSI compliant pneumatic brake while the second one is the blending according to tabulated TSI compliant limits.

\section{Line and electrical substations}

The electric line model is composed by a simple contact line model fed by electrical substations disposed throughout the line itself; the line supply the vehicle with the 


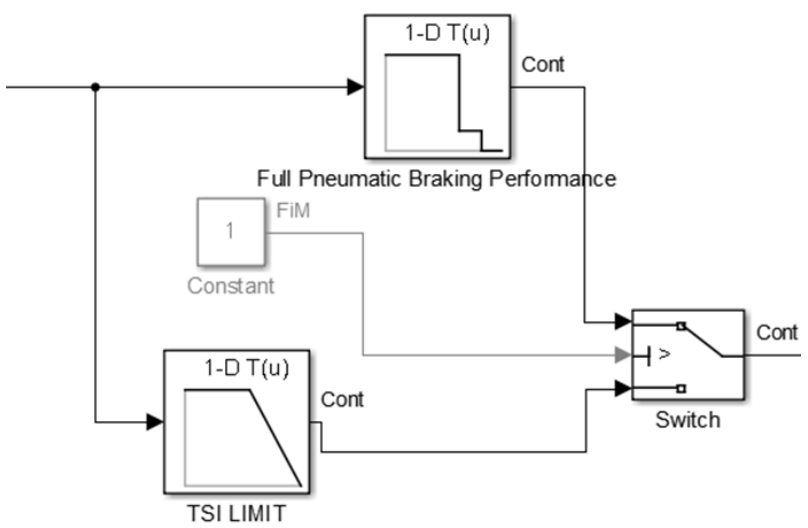

Figure 9: Blending strategy.

energy needed for its motion and it is also able to receive the energy produced by the vehicle during the regenerative braking phases. The energy due to those phases can be directly supplied to other accelerating vehicles present on the track or stored in energy storage devices placed in correspondence of some substations or on-board. During a dynamical simulation, the train position within the line varies and hence it modifies the line configuration; in particular, the line resistance between two vehicles or between a vehicle and the electrical substations varies with time (i.e. the left and right line resistances observed by the train as reported in Figure 6 are function of the vehicle position).

Those resistances can be calculated according to the following Equations:

$$
\left\{\begin{array}{l}
R_{\text {left }}=(1-\delta) \cdot R_{\text {tot }} \\
R_{\text {right }}=\delta \cdot R_{\text {tot }} \\
\delta=\frac{P_{2}-x}{L_{12}}
\end{array}\right.
$$

Where $\delta$ is the ratio between the distance of the train from the next substation $\left(P_{2}-x\right)$ and the distance between the two substations. These Equations are referred to the case of a bilateral power condition (i.e. when the vehicle moves between two substations). Figure 10 shows a scheme of the electrical balance on which the line model is based; in particular, the line node where the energy is fed to the vehicle is controlled by the following Equations:

$$
\begin{gathered}
i_{n-1}-i_{n}=i_{v}+i_{c} \\
V_{n+1}=V_{n}-R_{n} \cdot i_{n}
\end{gathered}
$$

Where $i_{n}$ is the current which leaves the $n$-th node, $V_{n}$ is the voltage, $i_{v}$ is current required by the vehicle, $i_{c}$ is the current due to an on-board storage devices and $R_{n}$ is the resistance of the correspondent line part. The current required by the vehicle during the traction phase (or equivalently, the current provided to the line during the 


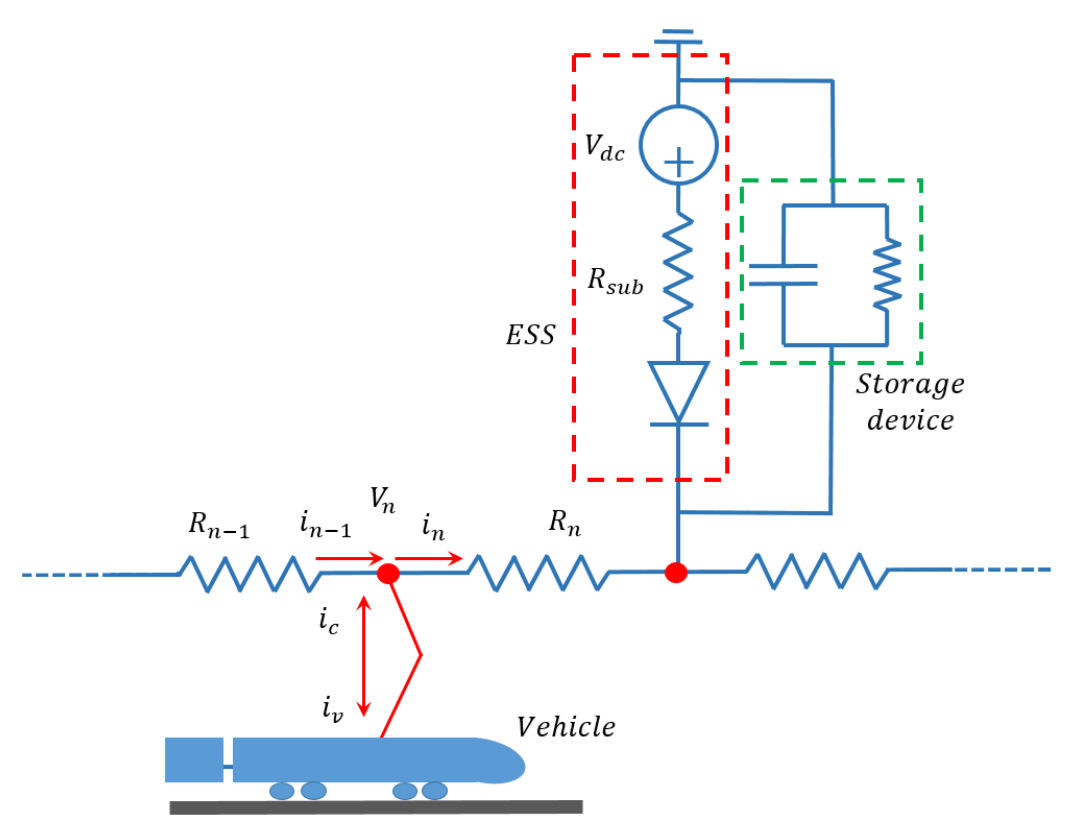

Figure 10: Scheme of the contact line model.

regenerative braking phases) can be calculated from the vehicle power, considering the efficiency of the electrical and mechanical components of the system:

$$
\begin{gathered}
P_{\text {traction }}=\frac{P_{\text {wheels }}}{\eta_{\text {gearbox }} \cdot \eta_{\text {motor }} \cdot \eta_{\text {motordrive }}} \\
P_{\text {braking }}=P_{\text {wheels }} \cdot \eta_{\text {gearbox }} \cdot \eta_{\text {motor }} \cdot \eta_{\text {motordrive }}
\end{gathered}
$$

The electrical substations main function is to feed the line approximating an ideal voltage source. They are often based on passive not reversible devices: a typical substation transform the alternate current provided by the grid into direct current to feed the vehicle. Thus the substation can be modelled as an ideal voltage source connected in series with a resistance (to represent the source internal voltage drop) and a diode. Furthermore it is also possible to consider the case of trains directly fed with the alternate current (i.e. like the high speed train considered in this paper for the validation of the model). The substation model can also include a stationary energy storage device. The node where the substation is connected to the contact line is represented by the following Equations:

$$
\begin{gathered}
V_{d} c-\left(i_{n}-i_{n-1}\right) \cdot R_{\text {sub }}=V_{n} \\
V_{n+1}=V_{n}-R_{n} \cdot i_{n},
\end{gathered}
$$

Where $V_{d c}$ is the voltage provided by the ideal voltage source and $R_{s u b}$ is the substation internal resistance. 


\section{Energy storage systems}

With the proposed model it is possible to consider the effect on the system of different types of storage devices. The most common stationary energy storage devices are batteries and supercapacitors: in this research activity, the authors propose the same model regardless of whether it is a battery or a supercapacitor (Figure 11).

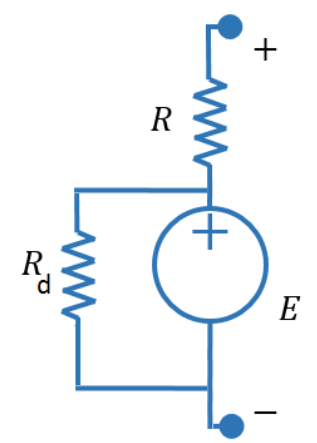

Figure 11: Scheme of the battery and supercapacitor model.

The proposed energy storage device model is composed by the following elementary elements:

- An ideal voltage generator $E$ (for the supercapacitor, the voltage is proportional to the integral of the current; while for the battery, the voltage is a non-linear function of the integral of the current);

- A resistance $R_{d}$ in parallel with the voltage source which simulates the device transient discharge;

- A resistance $R$ in series with the previous two elements.

This quite simple modelling approach allows to correctly simulate the behaviour of batteries and supercapacitors while requiring only a reduced set of parameters to work. Furthermore, this energy storage device, thanks to the modularity of the Simscape approach, can be easily connected in parallel with the electrical substations, allowing to simulate different scenarios and to search for the optimal spatial distribution of energy storage devices throughout the line: in fact, for each specific application it is possible to find the optimal distance between each stationary storage device in order to maximize the energy recovery and minimize the losses due the impedance of the line itself. Finally, the capacitor used as an on-board storage device can be modelled analogously to the stationary devices; this device can be used to store little amounts of energy (that can be useful in the case of tramways to go through short distances in absence of contact line) or to cut down voltage and current peaks. 


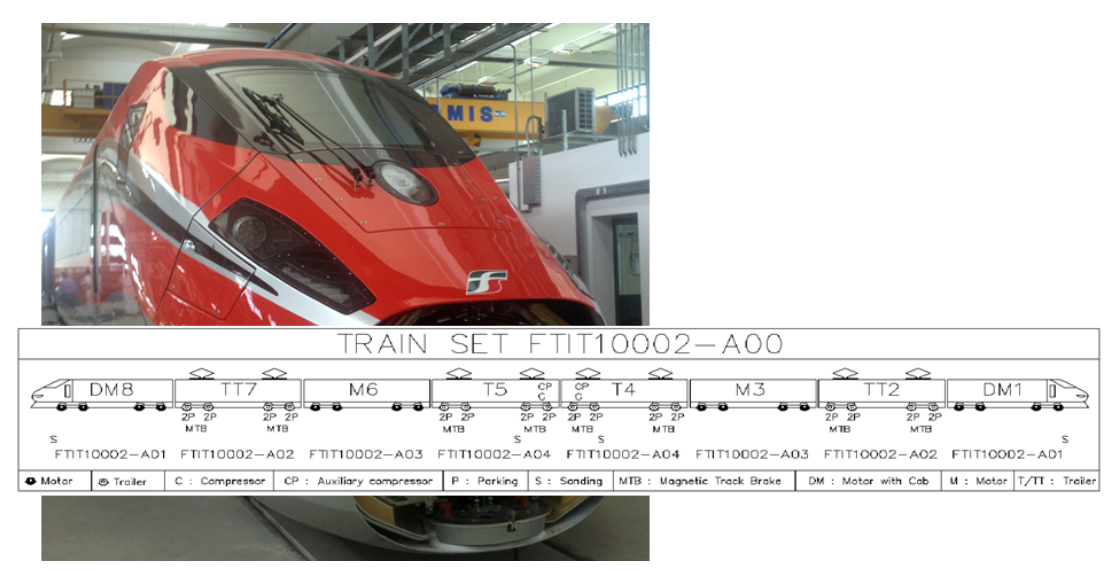

Figure 12: Test case.

\section{Experimental validation}

The proposed model has been tested and experimentally validated considering the characteristics of a new high speed train, the ETR 1000; the characteristics of the train which must be considered in the proposed model are reported in Figures 12, 8 and in Table 1. The model has been used to simulate four different scenarios, all of them

\begin{tabular}{|c|c|}
\hline Train mass & $495 \mathrm{t}$ \\
Motorized weight fraction & 0.5 \\
Nominal power & $9.8 \mathrm{MW}$ \\
Nominal speed & $300 \mathrm{~km} / \mathrm{h}$ \\
Brake pad 1 & $0.1-0.15 \mathrm{~cm}^{3} / \mathrm{MJ}$ \\
Brake pad 2 & $0.1-0.21 \mathrm{~cm}^{3} / \mathrm{MJ}$ \\
Line impedance & $0.05 \Omega / \mathrm{km}$ \\
ESS equivalent impedance & $0.1 \Omega$ \\
ESS no-load voltage & $3700 \mathrm{~V}$ \\
Mean distance between ESSs & $14.7 \mathrm{~km}$ \\
Max. distance between ESSs & $76 \mathrm{~km}$ \\
Min. distance between ESSs & $12 \mathrm{~km}$ \\
\hline
\end{tabular}

Table 1: Train and line characteristics.

simulated on the same line (representative of the Firenze-Roma line,as reported in Figure 13, where the position of the electrical substations and the slope of the lipne are shown) and with the same mission profile (i.e. a first acceleration, an $83 \mathrm{~m} / \mathrm{s}$ constant speed period and a final braking):

- A simulation without energy storage devices and hence without braking energy recovery;

- A simulation with supercapacitors connected to the electrical substations; 


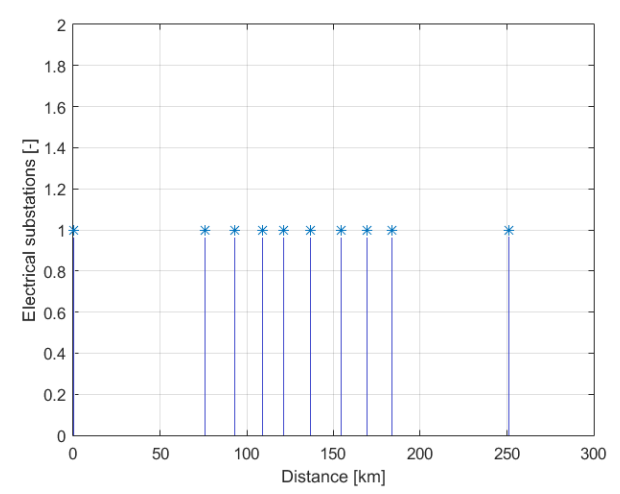

(a)

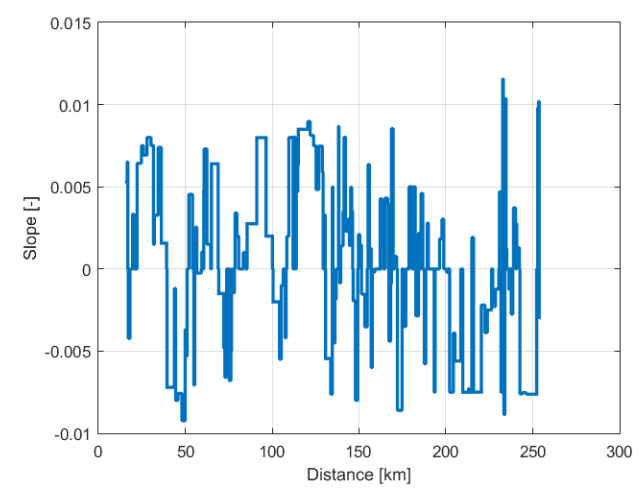

(b)

Figure 13: Scheme of the considered line: (a) substations and (b) slope.

- A simulation with batteries connected to the electrical substations;

- A simulation with batteries connected to the electrical substations and an onboard capacitor on the vehicle.

For the sake of synthesis, only the results referred to first basic case are reported in this paper, in order to highlight the global behavior of the model and its validation. First of all, Figure 14.a shows the velocity profile calculated with the proposed model: it is in good agreement with the chosen mission profile. The results shown in Figure 14 correspond to that velocity profile: it is possible to highlight how the higher power values corresponds to the initial acceleration phase to the maximum speed (Figure 14.b), analogously to the higher absorbed current (Figure 14.c) and to the bigger voltage drop (Figure 14.d). The other peaks and drops correspond to electrical substations and smaller accelerations due to the line characteristics; during the braking phases the voltage does not rise over the substation value because those results are referred to the first simulation scenario, the one without energy recovery.

Figure 15 shows the comparison between the results obtained with the proposed model and a set of experimental measurements obtained in a shorter part of the line: Figure 15.a shows the mission profile imposed to the vehicle to simulate the experimental case. Figures 15.b, 15.c and 15.d show respectively the power, the absorbed current and the voltage in correspondence of the vehicle pantograph: it is possible to highlight how the power increases up to the iso-power part of the vehicle traction characteristic (i.e. the velocity rises for the entire simulation) and how the current and the voltage show respectively a drop and a peak in correspondence of a substation placed in the considered line segment. The results obtained with the proposed model show a good agreement with the measured values. Figure 16.a shows the terms which contribute to the braking energy calculated considering different values of the requested braking effort: it is possible to highlight how the electrical braking gives an important contribute even considering a very simple mission profile (i.e. single final braking). The results shown in Figure 16.a highlight how the importance of the recovered brak- 


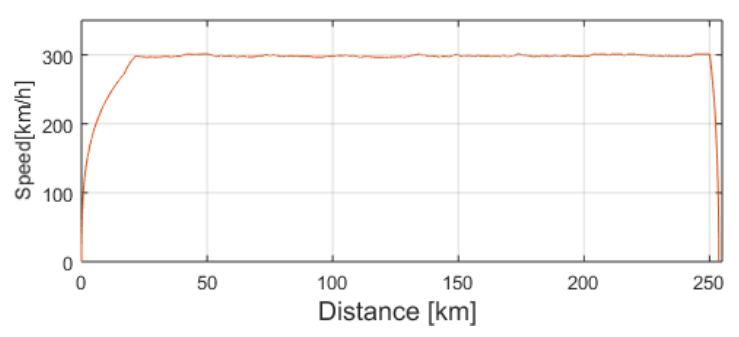

(a)

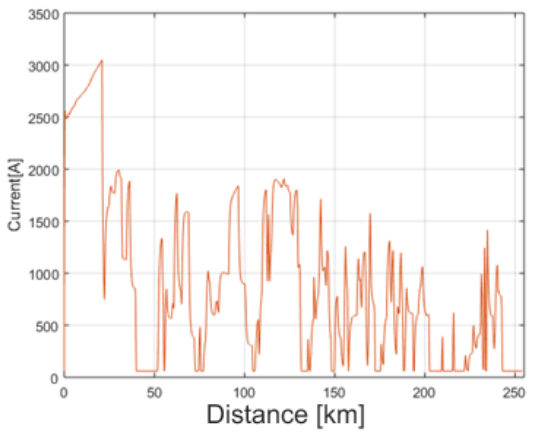

(c)

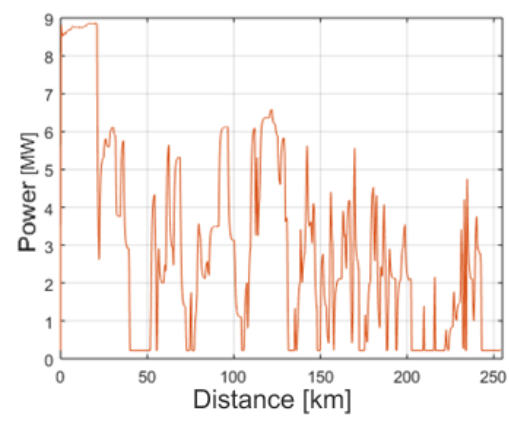

(b)

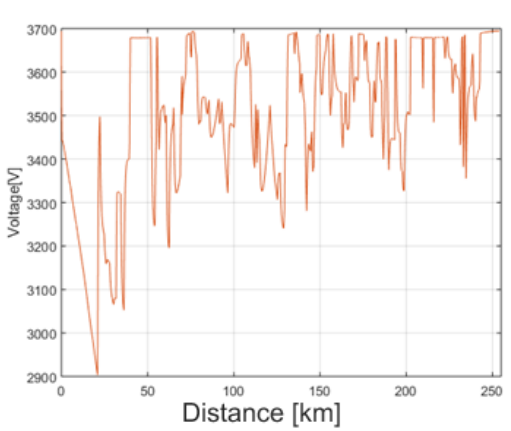

(d)

Figure 14: Preliminary results: (a) vehicle velocity profile, (b) power, (c) current and (d) voltage.

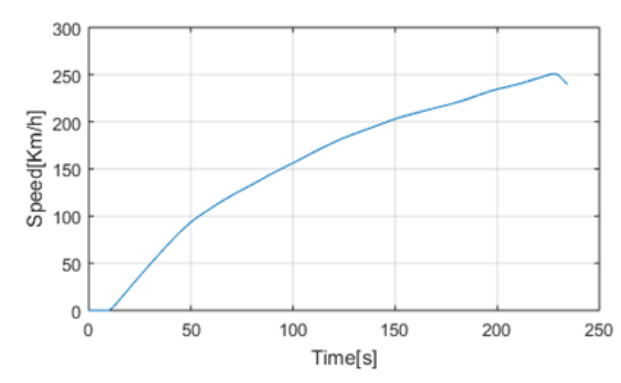

(a)

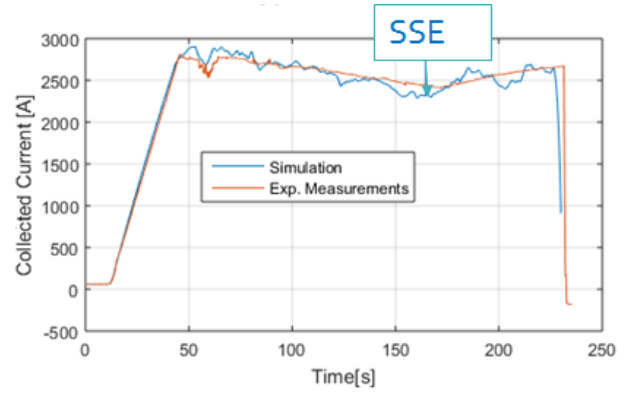

(c)

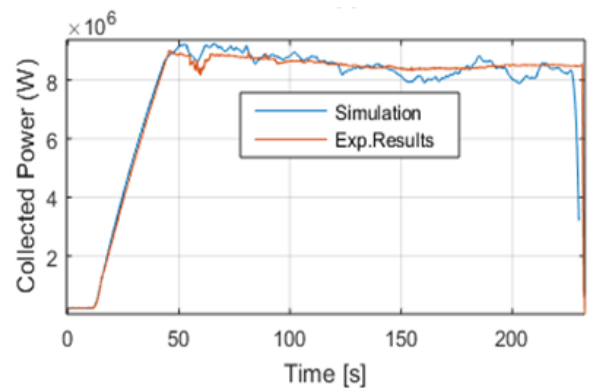

(b)

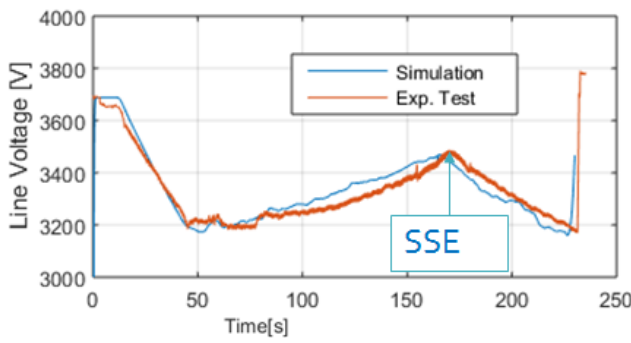

(d)

Figure 15: Comparison between the experimental measurements and the numerical results obtained with the proposed model. 


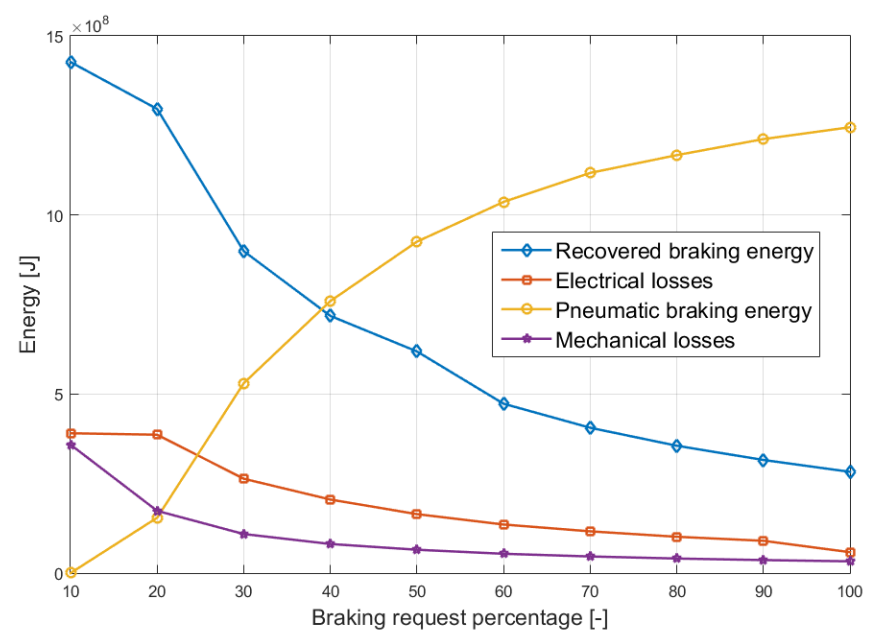

(a)

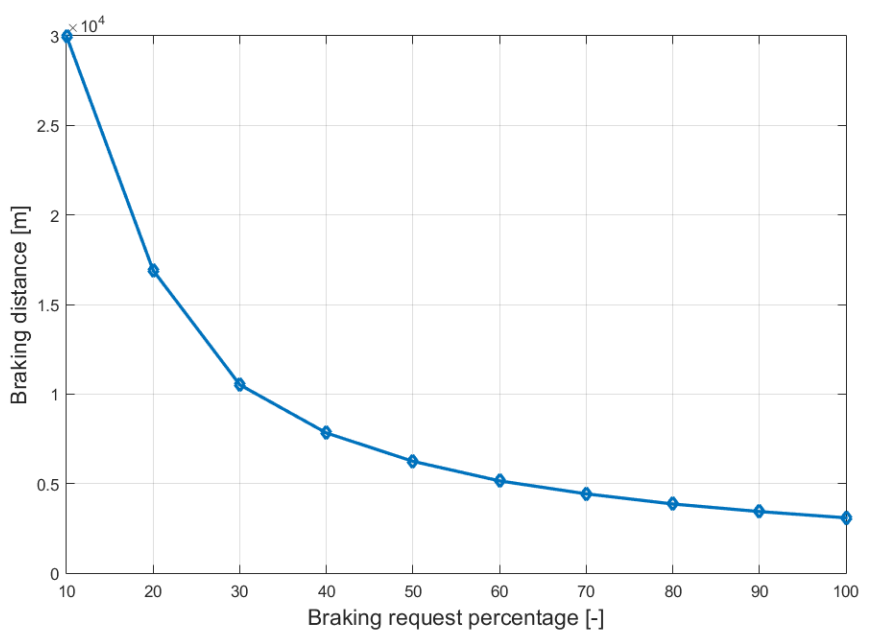

(b)

Figure 16: (a) Energy flows involved in the braking phase (from $83 \mathrm{~m} / \mathrm{s}$ to $0 \mathrm{~m} / \mathrm{s}$ ) and (b) braking distance with different braking efforts.

ing energy decreases increasing the requested braking effort and, consistently with this result, the pneumatic braking energy increases. Figure 16.b shows the braking distance with different braking efforts: the maximum values of the recovered braking energy corresponds to the higher values of the braking distance (i.e. when the applied braking effort is lower). Furthermore, it is possible to highlight how the energy dissipated due to mechanical losses is lower when the braking effort is higher (i.e. when the braking distance is lower).

Finally, Figure 17 shows the voltage in correspondence of the braking phase, considering different braking effort requests and different braking starting point between two adjacent electric substations: it is possible to highlight how the voltage peak is 


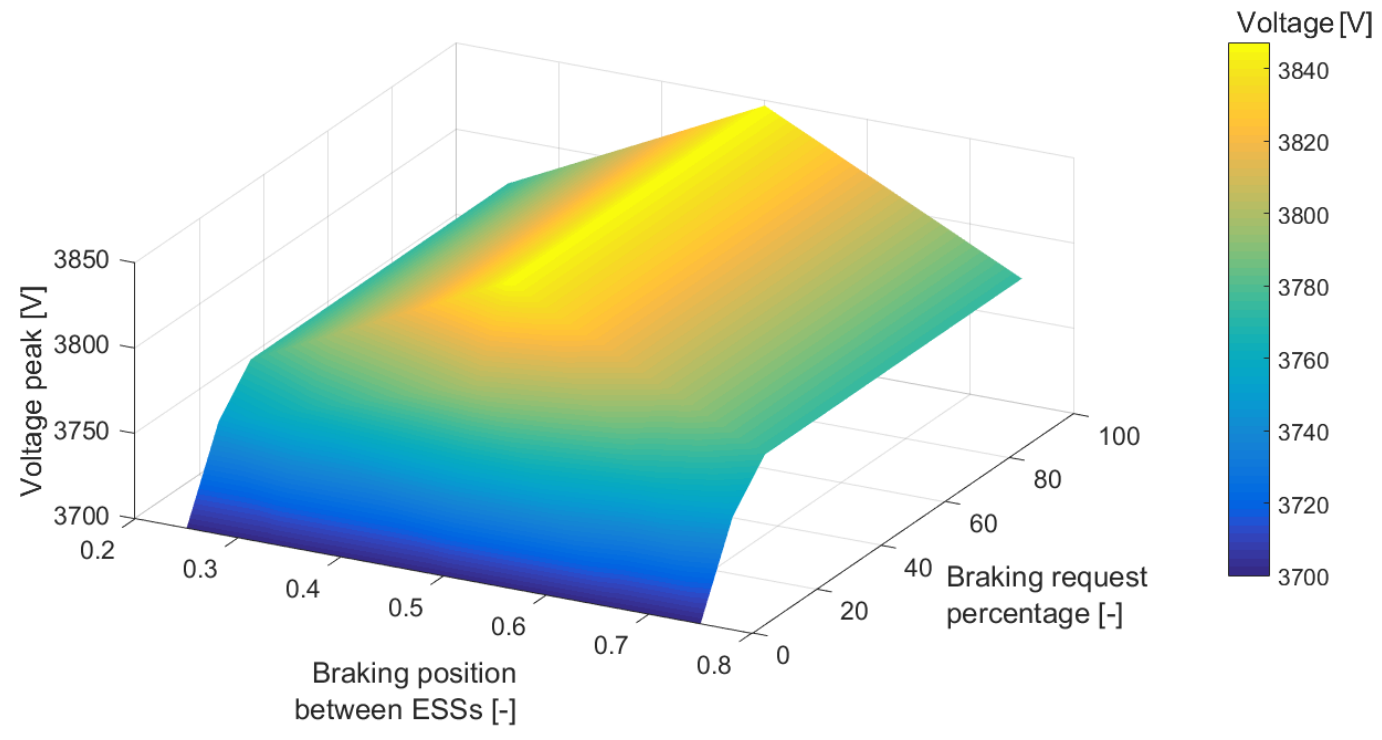

Figure 17: Braking voltage peak.

constant for braking requests higher than $20 \%$ and how the highest voltage values correspond to a braking phase which starts in the ESSs midspan.

\section{Conclusions}

In this work the authors presented a tool able to simulate and to optimize different aspects of the railway vehicle dynamics. With respect to previous works presented in literature, the tool is designed to integrate different modules able to simulate various physical phenomena (e.g. electrical and mechanical) which influence the optimization of energetic and efficiency aspects of the railway system. The proposed model has been developed and validated considering a benchmark case similar to a typical modern high speed train (e.g. the ETR 1000). The results of this analysis highlight two important aspects:

- From a modelling point of view, the proposed tool proved to be able to accurately reproduce the railway energetic aspects;

- From a physical point of view, the model highlights how energy storage systems can be useful even in high speed applications (i.e. applications where there is a small number of braking phases) in order to save energy and thus increase the sustainability of the railway system.

In particular, a simple stationary solution can be really effective in high speed train, while in tramways applications the use of on-board energy storage devices could be more worthy. In fact, for a high speed train the disadvantages due to an on-board energy storage device would higher, especially considering the weight of the device and the small number of braking phases, while a stationary solution allow to store easier 
the big but infrequent energy flows produced in the vehicle braking phase. Nowadays those results are widely acknowledged even by other research works by professional operators of the sector. In a short time, a more accurate investigation including the introduction of a more sophisticated management of energy storage systems through power converters and the simulation of more complex scenario with multiple convoys traveling on the same line will be realized. The use of the Matlab-Simscape language involves great advantages in terms of portability of the model between different Real Time targets, allowing for an easier use of the model in Hardware-In-The-Loop applications.

\section{References}

[1] EU Transport Pocketbook 2013.

[2] R. Conti, E. Galardi, E. Meli, D. Nocciolini, L. Pugi, A. Rindi Energy and wear optimisation of train longitudinal dynamics and of traction and braking systems, Vehicle System Dynamics Volume 53, Issue 5, May 2015, pages 651-671.

[3] Barrero R., Tackoen X., van Mierlo J., Stationary or onboard energy storage systems for energy consumption reduction in a metro network Proceedings of the Institution of Mechanical Engineers, Part F: Journal of Rail and Rapid Transit, May 1, 2010; vol. 224, pp. 207-225.

[4] Ceraolo M., Lutzemberger G., Stationary and on-board storage systems to enhance energy and cost efficiency of tramways, Journal of Power Sources, Volume 264, 15 October 2014, Pages 128-139, ISSN 0378-7753.

[5] Gonzlez-Gil A., Palacin R., Batty P., Powell J.P., A systems approach to reduce urban rail energy Consumption, Energy Conversion and Management, Volume 80, April 2014, Pages 509-524, ISSN 0196-8904.

[6] Gonzalez-Gil A., Palacin R., Batty P., Sustainable urban rail systems: Strategies and technologies for optimal management of regenerative braking energy, Energy Conversion and Management, Volume 75, November 2013, Pages 374-388, ISSN 0196-8904.

[7] Hillmansen S. and Roberts C., Energy storage devices in hybrid railway vehicles: A kinematic analysis, Proceedings of the Institution of Mechanical Engineers, Part F: Journal of Rail and Rapid Transit, January 1, 2007; vol. 221, pp. 135143.

[8] Pugi, L., Rindi, A., Ercole, A. G., Palazzolo, A., Auciello, J., Fioravanti, D., \& Ignesti, M. (2011). Preliminary studies concerning the application of different braking arrangements on Italian freight trains. Vehicle System Dynamics, 49(8), 1339-1365.

[9] L. Pugi, M. Malvezzi, S. Papini, G. Vettori, Design and preliminary validation of a tool for the simulation of train braking performance, Journal of Modern Transportation, 21, 247-257, 2013.

[10] E. Galardi, E. Meli, D. Nocciolini, L. Pugi, A. Rindi, Development of efficient models of Magnetic Braking Systems of railway vehicles, nternational Journal 
of Rail TransportationVolume 3, Issue 2, April 2015, pages 97-118.

[11] M. Ceraolo, G. Lutzemberger, and T. Huria. "Experimentally-determined models for high-power lithium batteries." Advanced Battery Technology (2011).

[12] E. Quaglietta, V. Punzo. "Supporting the design of railway systems by means of a Sobol variance-based sensitivity analysis.'Transportation Research Part C: Emerging Technologies 34 (2013): 38-54.

[13] Baccari S., Cammeo G., Dufour C., Iannelli L., Mungiguerra V., Porzio M., Reale G. and Vasca F., Real-Time Hardware-in-the-Loop in Railway: Simulations for Testing Control Software of Electromechanical Train Components, Railway Safety, Reliability, and Security: Technologies and Systems Engineering pp.221-248.

[14] UIC Fiche UIC 544-1 Brakes-Braking Power, 6th edition, October 2014.

[15] UIC Fiche UIC 541-3,Brakes - Disc brakes and their application - General conditions for the approval of brake pads Leaflet details 7 th edition, October 2010 Translation.

[16] EN 15734-1 Railway applications - Braking systems of high speed trains Part 1: Requirements and definitions.

[17] Official Web Site of E.R.A. http://www.era.europa.eu.

[18] Regulation 1302/2014 (1st merged RST TSI) EiF/DoA: 1/1/2015.

[19] Regulation 1301/2014 (1st merged ENE TSI) EiF/DoA: 1/1/2015 gulation 1301/2014 (1st merged ENE TSI) EiF/DoA: 1/1/2015.

[20] Regulation 321/2013 (2nd CR WAG TSI) EiF 13/4/2013 DoA: 1/1/2014.

[21] Breedveld, Peter C., Ronald C. Rosenberg, and T. Zhou. "Bibliography of bond graph theory and application." Journal of the Franklin Institute 328, no. 5-6 (1991): 1067-1109.

[22] Widl, Edmund, Peter Palensky, and Atiyah Elsheikh. "Evaluation of two approaches for simulating cyber-physical energy systems." In IECON 2012-38th Annual Conference on IEEE Industrial Electronics Society, pp. 3582-3587. IEEE, 2012.

[23] L. Pugi, R. Conti, D. Nocciolini, E. Galardi, A. Rindi, S. Rossin (2014). A Tool for the Simulation of Turbo-Machine Auxiliary Lubrication Plants. International Journal of Fluid Power, vol. 2-15, p. 87-100, ISSN: 1439-9776, doi: 10.1080/14399776.2014.931130. 